\title{
ROJ
}

\section{Value of imaging study in predicting pelvic lymph node metastases of uterine cervical cancer}

\author{
Wonguen Jung, MD¹, Kyung Ran Park, MD, PhD¹, Kyung-Ja Lee, MD, PhD1, Kyubo Kim, MD, PhD1, \\ Jihae Lee, MD1', Songmi Jeong, MD, PhD'1, Yi-Jun Kim, MD¹, Jiyoung Kim, MD¹, Hai-Jeon Yoon, MD², \\ Byung-Chul Kang, MD, PhD ${ }^{3}$, Hae Soo Koo, MD, PhD ${ }^{4}$, Sun Hee Sung, MD, PhD ${ }^{4}$, Min-Sun Cho, MD, PhD ${ }^{4}$, \\ Sanghui Park, MD, PhD ${ }^{4}$
}

Departments of ${ }^{1}$ Radiation Oncology, ${ }^{2}$ Nuclear Medicine, ${ }^{3}$ Radiology, and ${ }^{4}$ Pathology, Ewha Womans University Mokdong Hospital, Ewha Womans University College of Medicine, Seoul, Korea

Purpose: To evaluate the diagnostic accuracy of computed tomography (CT), magnetic resonance imaging (MRI), and positron emission tomography/computed tomography (PET/CT) in predicting pelvic lymph node (LN) metastases in patients with cervical cancer.

Materials and Methods: From January 2009 to March 2015, 114 patients with FIGO stage IA1-IIB uterine cervical cancer who underwent hysterectomy with pelvic lymphadenectomy and took CT, MRI, and PET/CT before surgery were enrolled in this study. The criteria for $L N$ metastases were a $L N$ diameter $\geq 1.0 \mathrm{~cm}$ and/or the presence of central necrosis on $C T$, a $L N$ diameter $\geq 1.0 \mathrm{~cm}$ on MRI, and a focally increased FDG uptake on PET/CT. The sensitivity, specificity, positive predictive value (PPV), negative predictive value (NPV), and accuracy for pelvic LN metastases were estimated.

Results: The sensitivity, specificity, PPV, NPV, and accuracy for detection of pelvic LN metastases were $51.4 \%, 85.9 \%, 41.3 \%$, 90.1\%, and $80.3 \%$ for CT; $24.3 \%, 96.3 \%, 56.3 \%, 86.8 \%$, and $84.6 \%$ for MRI; and $48.6 \%, 89.5 \%, 47.4 \%, 90.0 \%$, and $82.9 \%$ for PET/ $C T$, respectively. The sensitivity of PET/CT and CT was higher than that of MRI ( $p=0.004$ and $p=0.013$, respectively). The specificity of MRI was higher than those of PET/CT and CT ( $p=0.002$ and $p=0.001$, respectively). The difference of specificity between PET/CT and CT was not statistically significant $(p=0.167)$.

Conclusion: These results indicate that preoperative CT, MRI, and PET/CT showed low to moderate sensitivity and PPV, and moderate to high specificity, NPV, and accuracy. More efforts are necessary to improve sensitivity of imaging modalities in order to predict pelvic LN metastases.

Keywords: Uterine cervical neoplasms, Lymph nodes, Magnetic resonance imaging, Computed tomography, Positron-emission tomography

\section{Introduction}

Uterine cervical cancer is one of the most common cancers in women worldwide. The incidence of uterine cervical cancer in Korea has decreased, but it ranks still fifth among cancers in women [1]. Rather, the number of patients who received

Received 17 April 2017, Revised 25 July 2017, Accepted 14 August 2017.

Correspondence: Kyung Ran Park, MD, PhD and Kyubo Kim, MD, PhD, Department of Radiation Oncology, Ewha Womans University College of Medicine, 1071 Anyangcheon-ro, Yangcheon-gu, Seoul 07985, Korea. Tel: +82-2-2650-5334, Fax: +822-2654-0363, E-mail: krparkia@gmail.com, kyubokim.ro@gmail.com

(c) This is an Open Access article distributed under the terms of the Creative Commons Attribution Non-Commercial License (http://creativecommons.org/ licenses/by-nc/4.0/) which permits unrestricted non-commercial use, distribution, and reproduction in any medium, provided the original work is properly cited.

www.e-roj.org 
radiotherapy for uterine cervical cancer is increasing [2]. Pelvic lymph node (LN) involvement is a significant prognostic factor for overall survival $[3,4]$ and is one of the most important indications for adjuvant concurrent chemoradiotherapy (CCRT) or definitive CCRT in patients with cervical cancer. Thus, accurate pretherapeutic evaluation of pelvic LN metastases is critical for appropriate treatment selection and planning. The boost external beam radiotherapy (EBRT) to clinically positive pelvic LNs could achieve favorable outcomes in pelvic nodal control in patients with uterine cervical cancer treated by CCRT or radiotherapy [5]. Although the boost EBRT improved pelvic nodal control, the normal tissue to the surrounding pelvic LNs was simultaneously irradiated. Thus, it is important to administer the boost EBRT in selected patients with accurate assessment of clinically positive $L N$ to reduce the volume of irradiated normal tissue and to minimize the complication rates. The utility of imaging modalities in RT planning would improve the selection of patients receiving the boost EBRT. Surgical pelvic LN evaluation is the gold standard for the diagnosis of pelvic LN metastases. However, pelvic lymphadenectomy increases the risk of acute and late complications such as lymphedema, infection, vascular damage and ureteral injury to the patients. Therefore, a non-invasive technique for precisely identifying LN status is necessary not only to avoid unnecessary surgical procedures but also to serve as a basis for optimal radiotherapy field and dose without surgery $[6,7]$. Several non-invasive modalities have been used to assess the status of the disease; computed tomography (CT), magnetic resonance imaging (MRI) and positron emission tomography/computed tomography (PET/CT).

The aim of this study was to investigate the diagnostic accuracy of $C T, M R I$, and $P E T / C T$ in predicting pelvic $L N$ metastases by comparing imaging findings with histopathologic diagnosis after LN dissection in patients with uterine cervical cancer.

\section{Materials and Methods}

\section{Study population}

Eligibility requirements for the current study included newly diagnosed and histopathologically proven, the International Federation of Gynecology and Obstetrics (FIGO) stage IA1-IIB uterine cervical carcinoma, as determined by baseline study including CT, MRI, and PET/CT from January 2009 to March 2015. All patients who underwent hysterectomy and bilateral pelvic lymphadenectomy were eligible to participate in this study. The patients had no contraindications for surgery and had no clinical evidence of distant metastases. The patients who took PET alone were excluded from this study, because PET scan has limited resolution and anatomic information compared with PET/CT. This research protocol was approved by the Institutional Review Board of Ewha Womans University Mokdong Hospital (No. 2015-08-029).

\section{CT}

CT examinations were performed using CT scans (SOMATOM; Siemens Medical Solutions, Erlangen, Germany), with the following parameters: beam collimation, $0.6 \mathrm{~mm} \times 128 \mathrm{~mm}$; pitch, 0.8 ; gantry rotation time, 0.5 seconds; slice thickness, $5.0 \mathrm{~mm}$; interval, $5.0 \mathrm{~mm}$. For the contrast enhancement study, $120 \mathrm{~mL}$ of non-ionic contrast medium (iohexol, Bonorex 350; Centrial Medical Service, Seoul, Korea) was intravenously administered at a rate of $3.0 \mathrm{~mL} / \mathrm{s}$. Then, CT scan was performed 100 seconds after contrast material injection to obtain the venous phase images. All images were obtained from the abdomen to the pelvis. The CT images were interpreted by a radiologist in gynecologic oncoradiology with being unware of the results of other tests. LNs with the short axis diameter $\geq 1.0 \mathrm{~cm}$ and/or the appearance of central necrosis were considered positive for malignancy on CT images.

\section{MRI}

MR imaging of the abdomen and pelvis was obtained by using 3.0-T scanner (Achieva; Philips Medical Systems, Cleveland, $\mathrm{OH}, \mathrm{USA}$ ) using a pelvic array coil for pelvic scans. Scans were performed using the following parameters: axial T1-weighted fast spin-echo (FSE) sequence (repetition time [TR]/echo time [TE], $500 \mathrm{~ms} / 10 \mathrm{~ms}$; echo-train length, 4; field-of-view [FOV], $24 \mathrm{~cm} \times 24 \mathrm{~cm}$; matrix, $512 \times 248$; section thickness, $4.0 \mathrm{~mm}$; no intersectional gap; three signals acquired; no fat saturation; bandwidth, $0.291 \mathrm{kHz}$ ), axial T2-weighted FSE sequence (TR/ TE, 3105 ms/90 ms; echo train length, 19; FOV, $24 \mathrm{~cm} \times 24 \mathrm{~cm}$; matrix, $552 \times 239$; section thickness, $4.0 \mathrm{~mm}$; no intersectional gap; four signals acquired; no fat saturation, bandwidth, 0.190 $\mathrm{kHz}$ ), sagittal T2-weighted FSE sequence (TR/TE, 3,000 ms/90 ms; echo train length, 19; FOV, $24 \mathrm{~cm} \times 24 \mathrm{~cm}$; matrix, $520 \times$ 247; section thickness, $3.0 \mathrm{~mm}$; intersectional gap, $0.3 \mathrm{~mm}$; four signals acquired; no fat saturation, bandwidth, 0.179 $\mathrm{kHz})$, coronal T2-weighted FSE sequence (TR/TE, 2,150 ms/70 ms; echo train length, 12; FOV, $24 \mathrm{~cm} \times 24 \mathrm{~cm}$; matrix, 520 $\times$ 240; section thickness, $3.0 \mathrm{~mm}$; no intersectional gap; four signals acquired; no fat saturation, bandwidth, $0.138 \mathrm{kHz}$. After the acquisition of these images, diffusion-weighted MRI 
(DW-MRI) was obtained in the sagittal plane using a singleshot echo-planar imaging sequence (TR/TE, 5,000 ms/60 ms; flip angle, $90^{\circ}$; three excitations; matrix, $100 \times 100$, bandwidth, $0.2 \mathrm{kHz}$ ) with chemical shift selective (CHESS) fat suppression technique. The corresponding $b$-values to the diffusion sensitizing gradient were 0 and $1,000 \mathrm{~s} / \mathrm{mm}^{2}$. An apparent diffusion coefficient (ADC) maps were automatically calculated on a pixel-by-pixel basis from the DW-MRI according to the following equation:

$$
A D C=\frac{\ln (S 1)-\ln (S 0)}{b 1-b 0}
$$

where b0 and b1 represent lower and higher b-values, respectively, and S0 and S1 are the signal intensities for DW$\mathrm{MRI}$ in these b-values. Forty patients did not undergo DW-MRI because they were recruited between January 2011 and June 2012, during which time we did not perform DW-MRI. The $\mathrm{MRI}$ images were interpreted by a radiologist in gynecologic oncoradiology with being unware of the results of other tests. LNs with the short axis diameter $\geq 1.0 \mathrm{~cm}$ were considered positive for malignancy on MR images.

\section{4. ${ }^{18}$ F-FDG-PET/CT}

PET/CT scanning was obtained using a whole-body PET system (Biograph 128; Siemens Medical Solutions) by the use of ${ }^{18} \mathrm{~F}$-FDG. The patients were asked to empty their bladder before administration of ${ }^{18} \mathrm{~F}-\mathrm{FDG}$ and over again before image obtaining. After an intravenous injection of 3.0 to $5.0 \mathrm{MBq} /$ $\mathrm{kg}$ of ${ }^{18} \mathrm{~F}$-FDG followed by an uptake phase of 60 minutes, PET/CT scanning of the whole body was performed. The nonenhanced CT scans were initially performed with the following parameters: FOV, skull base to the thigh; tube voltage, $80 \mathrm{kV}$; automatic mA/s adjustment (CARE Dose 4D; Siemens Medical Solutions); section thickness, $3.0 \mathrm{~mm}$; pitch, 1.2. PET scans were performed immediately after CT. The spatial resolution of PET was $2.0 \mathrm{~mm}$ at the full width at half maximum (FWHM) in the transaxial direction and $2.0 \mathrm{~mm}$ FWHM in the axial direction at the center. Whole-body PET images (skull to midthigh) were obtained with 3D emission scan and acquired at 2 minutes per bed position. PET images were reconstructed to $200 \times 200$ matrices, $3.4 \mathrm{~mm} \times 3.4 \mathrm{~mm}$ pixel size, and 3.0 $\mathrm{mm}$ section thickness with the TrueX algorithm (3 iterations, 21 subsets) with point spread function and time of flight. The PET/CT images were interpreted by a nuclear medicine physician with being unware of the results of other tests. For each patient, FDG uptakes by normal tissue and tumor were measured as a value of uptake counts. On PET/CT images, a malignant LN was defined as a focally increased FDG uptake greater than the maximum standardized uptake values $\left(S U V_{\text {max }}\right)$ 3.0 or moderately to markedly increased FDG uptake relative to the surrounding tissue with the exclusion of urinary activity, vessel and physiological bowel. Equivocal or unclear uptakes were interpreted as negative findings.

\section{Histopathologic evaluation}

LNs were labeled as right and left pelvis. All LNs were cut at 2.0 $\mathrm{mm}$ thickness and stained with hematoxylin and eosin. Each LN was examined by pathologists, who were blinded to the imaging results and surgical exploration findings.

\section{Classification of lymph node}

The pelvic LNs were divided into two groups: right hemi-pelvis and left hemi-pelvis. The hemi-pelvis based classification was applied to the current study as well as several other studies of uterine neoplasms $[8,9]$. The right hemi-pelvic LNs included right common iliac area, right internal iliac area, right external iliac area, and right obturator area. The left hemi-pelvic LNs included left common iliac area, left internal iliac area, left external iliac area, and left obturator area.

\section{Statistical evaluation}

$C T, M R I$, and PET/CT images on pelvic LN metastases were estimated on the basis of the histologic examination as true positive, false positive, true negative, or false negative. The pelvic LNs detected by imaging were correlated with the histologic findings on each hemi-pelvis as a distinct unit Findings were considered a true positive if the positive pelvic LNs were shown in one hemi-pelvis on $\mathrm{CT}, \mathrm{MRI}$ or PET/CT imaging and if metastatic LNs were found per hemi-pelvis on pathology. Findings were considered a false positive if the positive pelvic LNs were shown in one hemi-pelvis on CT, MRI or PET/CT imaging and if no metastatic LNs were identified per hemi-pelvis on pathology. Findings were considered a true negative if all LNs were free of metastases per hemi-pelvis on both imaging and pathology. Findings were considered a false negative if the positive pelvic LNs were not shown in one hemi-pelvis on $\mathrm{CT}, \mathrm{MRI}$ or PET/CT imaging and if metastatic LNs were found per hemi-pelvis on pathology. The sensitivity, specificity, positive predictive value (PPV), negative predictive value (NPV), and accuracy of the CT, MRI and PET/CT for detection of pelvic $\mathrm{LN}$ involvement were estimated using standard statistical formulas. The relative sensitivities and specificities of $C T, M R I$, and PET/CT in detecting LN metastases were compared by using the McNemar's test (SPSS software 
Table 1. The distribution of enrolled patients and rate of positive pelvic LNs on surgery according to the FIGO stage

\begin{tabular}{ccc}
\hline FIG0 stage & $\begin{array}{c}\text { No. of patients } \\
(\%)\end{array}$ & $\begin{array}{c}\text { No. of patients with positive } \\
\text { pelvic LNs on surgery }(\%)\end{array}$ \\
\hline IA & $9(7.9)$ & $0(0)$ \\
IA1 & $5(4.4)$ & $0(0)$ \\
IA2 & $4(3.5)$ & $0(0)$ \\
IB & $88(77.2)$ & $15(17.0)$ \\
IB1 & $77(67.5)$ & $13(16.9)$ \\
IB2 & $11(9.7)$ & $2(18.2)$ \\
IIA & $7(6.1)$ & $2(28.6)$ \\
IIA1 & $3(2.6)$ & $0(0)$ \\
IIA2 & $4(3.5)$ & $2(50.0)$ \\
IIB & $10(8.8)$ & $6(60.0)$ \\
Total & 114 & $23(20.2)$ \\
\hline
\end{tabular}

LN, lymph node; FIGO, International Federation of Gynecology and Obstetrics.

Table 2. Histopathologic characteristics of pelvic LNs

\begin{tabular}{lcc}
\hline \multicolumn{1}{c}{ Characteristic } & $\begin{array}{c}\text { Right } \\
\text { hemi-pelvis }\end{array}$ & $\begin{array}{c}\text { Left } \\
\text { hemi-pelvis }\end{array}$ \\
\hline LNs harvested per patient & $16.9(5-36)$ & $16.4(4-42)$ \\
Total LNs harvested & 1,923 & 1,871 \\
Total metastatic LNs harvested & 36 & 30 \\
Patients with pathologic LNs & 23 & 14 \\
metastases & & \\
\hline
\end{tabular}

Values are presented as mean (range) or number.

LN, lymph node.

ver. 18.0; SPSS Inc., Chicago, IL, USA). A level of $p$-value $<0.05$ was considered as statistically significant.

\section{Results}

A total of 114 patients were enrolled. The distribution of enrolled patients and rate of positive pelvic LN on surgery according to the 2009 FIGO stage were shown in Table 1. Median age was 48 years (range, 26 to 86 years). For 114 patients, 228 (right, left) sites of pelvic LN were analyzed. The histopathologic characteristics of pelvic LNs were shown in Table 2. Pelvic LN metastasis was present in 37 sites among 228 pelvic LN sites (16.2\%) on surgical specimens. CT, MRI, and PET/CT detection profiles of LN sites were shown in Table 3. The positive findings of LN metastases in CT, MRI, and PET/ CT were present in 46 (20.2\%), 16 (7.0\%), and 38 (16.7\%) LN sites, respectively. Seven of the 37 pelvic LN sites showed positive findings of $\mathrm{LN}$ metastases in $\mathrm{CT}, \mathrm{MRI}$, and PET/CT and pathology (Fig. 1). Five of the 37 pelvic LN sites showed positive findings in all three imaging modalities but no metastatic LNs on pathology (Fig. 2). For 38 sites with positive findings on PET/CT, the SUV $V_{\max }$ of pelvic LNs ranged from 1.3 to 7.4 , with a mean value of 3.1. The SUV $V_{\max }$ of pelvic LNs with and without pathologic metastases were $3.3 \pm 1.8(1.3-7.4)$ and $3.0 \pm 0.7(1.7-4.5)$, respectively (Student t-test, $p=0.42$ ).

The hemi-pelvic LN-based sensitivity, specificity, PPV, NPV, and accuracy of CT, MRI, and PET/CT for predicting LN metastasis were shown in Table 4. As for specificity, PPV, and accuracy, MRI had the greatest values among three imaging modalities for detection of metastatic LN. Regarding sensitivity and NPV, CT had the greatest values. The sensitivity of CT was higher than those of MRI or PET/CT. The differences of sensitivity were statistically significant between CT and MRI $(p=0.013)$ as well as PET/CT and MRI $(p=0.004)$, respectively. The difference of sensitivity between CT and PET/CT was not statistically significant $(p=1.000)$. The specificity of MRI was higher than those of CT or PET/CT. The differences of specificity were statistically significant between CT and MRI $(p<0.001)$ as well as PET/CT and MRI $(p=0.002)$, respectively. The difference of specificity between PET/CT and CT was not statistically significant $(p=0.167)$.

The sensitivity and specificity of patients with FIGO IA and

Table 3. CT, MRI, and PET/CT detection profiles of LNs according to hemi-pelvis

\begin{tabular}{|c|c|c|c|}
\hline \multirow{2}{*}{ Modality } & \multicolumn{3}{|c|}{ Clinically positive LNs } \\
\hline & $\begin{array}{l}\text { Right hemi-pelvis } \\
\qquad(\mathrm{n}=114)\end{array}$ & $\begin{array}{l}\text { Left hemi-pelvis } \\
\qquad(n=114)\end{array}$ & Total \\
\hline CT & 24 & 22 & 46 \\
\hline MRI & 9 & 7 & 16 \\
\hline PET/CT & 20 & 18 & 38 \\
\hline Pathology & 23 & 14 & 37 \\
\hline
\end{tabular}

CT, computed tomography; MRI, magnetic resonance imaging; PET/CT, positron emission tomography/computed tomography; LN, lymph node. 

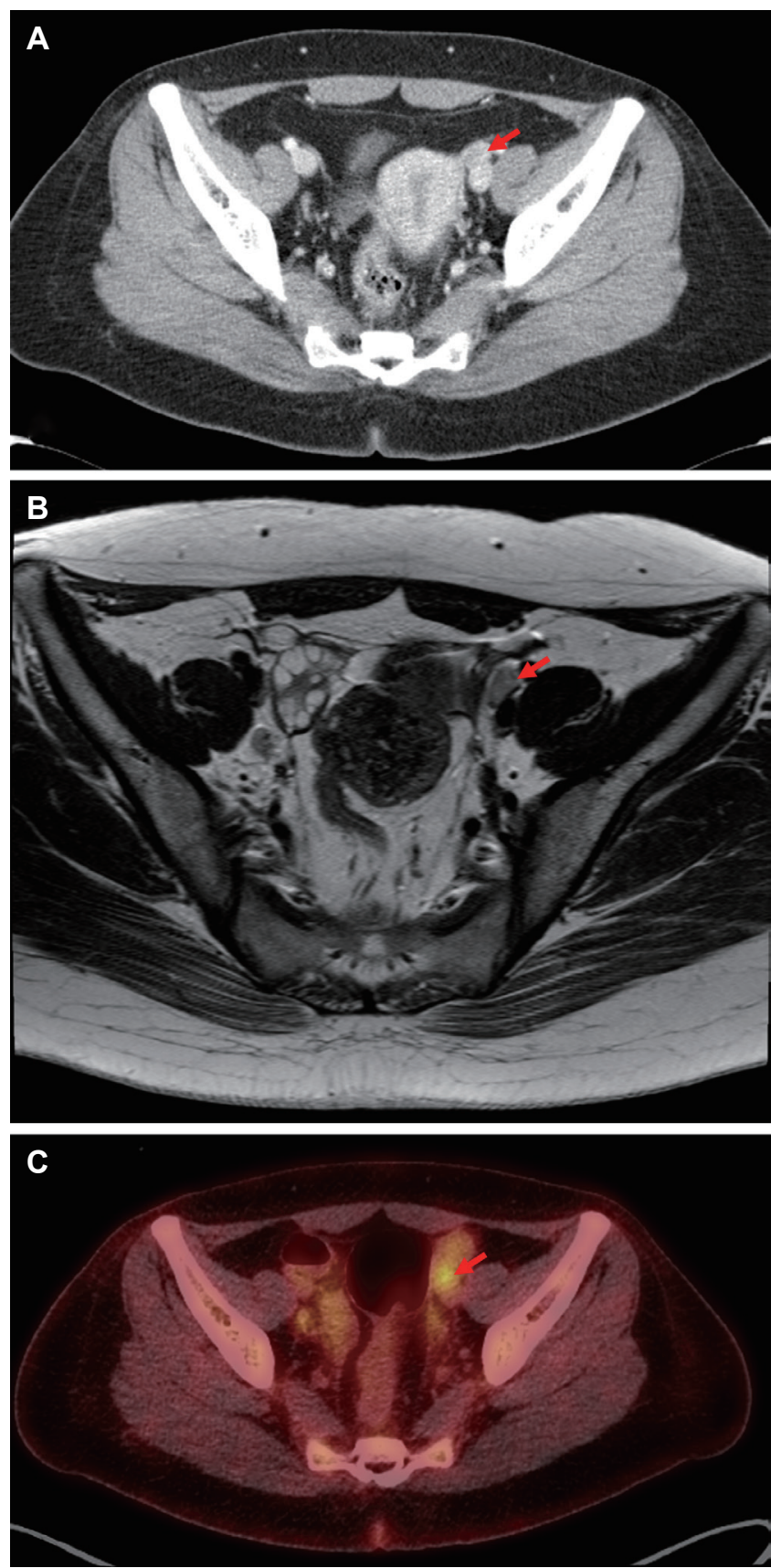

Fig. 1. Corresponding CT (A), MRI (B), and PET/CT (C) images in a patient with true positive pelvic lymph node (LN). (A) Enhanced CT image showed an enlarged pelvic LN (arrow) in left external iliac area. (B) T2-weighted MR image showed an enlarged pelvic LN (arrow) in left external iliac area. (C) PET/CT scan showed that increased FDG uptake (arrow) corresponds to the enlarged left external iliac LN seen in CT and MRI, suggesting LN metastasis. Histopathologic examination confirmed LN involvement. CT, computed tomography; MRI, magnetic resonance imaging; PET/ CT, positron emission tomography/computed tomography; FDG, fludeoxyglucose.
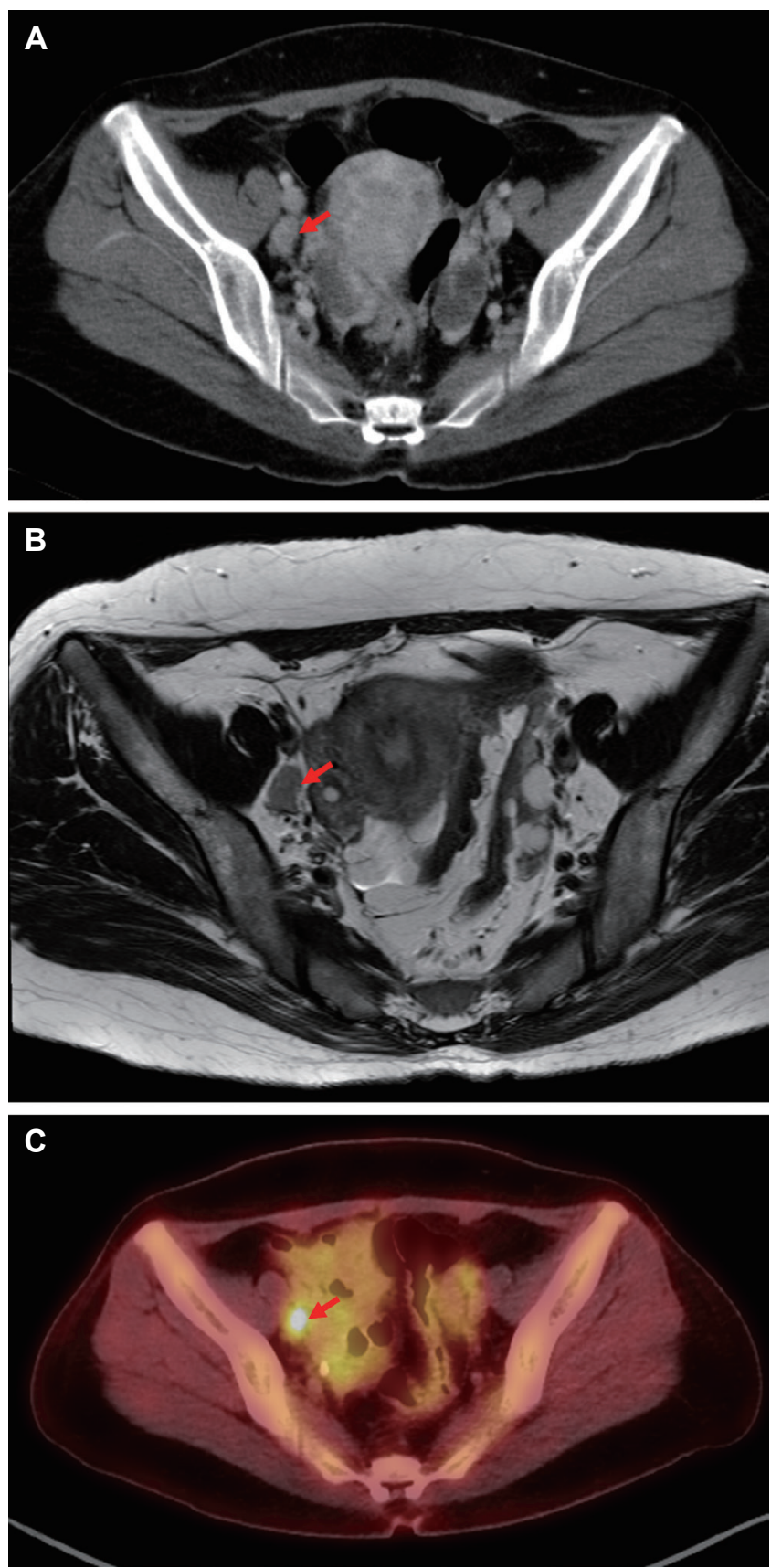

Fig. 2. Corresponding CT (A), MRI (B), and PET/CT (C) images in a patient with false positive pelvic lymph node (LN). (A) Enhanced CT image showed an enlarged pelvic LN (arrow) in right external iliac area. (B) T2-weighted MR image showed an enlarged pelvic LN (arrow) in right external iliac area. (C) PET/CT scan showed that increased FDG uptake (arrow) corresponds to the enlarged right external iliac $L N$ seen in $C T$ and $M R I$, suggesting $L N$ metastasis. Histopathologic examination confirmed no LN involvement. CT, computed tomography; MRI, magnetic resonance imaging; PET/ $C T$, positron emission tomography/computed tomography; FDG, fludeoxyglucose. 
Table 4. Comparison of findings of CT, MRI, and PET/CT with histologic findings based on the LN sites

\begin{tabular}{|c|c|c|c|c|c|c|c|c|c|}
\hline \multirow{2}{*}{ Imaging modality } & \multicolumn{4}{|c|}{ Histologic findings (No. of sites) } & \multirow{2}{*}{$\begin{array}{c}\text { Sensitivity } \\
(\%)\end{array}$} & \multirow{2}{*}{$\begin{array}{c}\text { Specificity } \\
(\%)\end{array}$} & \multirow{2}{*}{$\begin{array}{l}\text { PPV } \\
(\%)\end{array}$} & \multirow{2}{*}{$\begin{array}{l}\text { NPV } \\
(\%)\end{array}$} & \multirow{2}{*}{$\begin{array}{c}\text { Accuracy } \\
(\%)\end{array}$} \\
\hline & TP & $\mathrm{FP}$ & TN & FN & & & & & \\
\hline \multicolumn{10}{|l|}{ Overall $(n=228)$} \\
\hline CT & 19 & 27 & 164 & 18 & 51.4 & 85.9 & 41.3 & 90.1 & 80.3 \\
\hline MRI & 9 & 7 & 184 & 28 & 24.3 & 96.3 & 56.3 & 86.8 & 84.6 \\
\hline $\mathrm{PET} / \mathrm{CT}$ & 18 & 20 & 171 & 19 & 48.6 & 89.5 & 47.4 & 90.0 & 82.9 \\
\hline \multicolumn{10}{|l|}{ FIGO IA and IB $(n=194)$} \\
\hline CT & 9 & 19 & 151 & 15 & 37.5 & 88.8 & 32.1 & 91.0 & 82.5 \\
\hline MRI & 4 & 5 & 165 & 20 & 16.7 & 97.1 & 44.4 & 89.2 & 87.1 \\
\hline PET/CT & 10 & 14 & 156 & 14 & 41.7 & 91.8 & 41.7 & 91.8 & 85.6 \\
\hline \multicolumn{10}{|l|}{ FIGO IIA and IIB $(n=34)$} \\
\hline $\mathrm{CT}$ & 10 & 8 & 13 & 3 & 76.9 & 61.9 & 55.6 & 81.3 & 67.6 \\
\hline MRI & 5 & 2 & 19 & 8 & 38.5 & 90.5 & 71.4 & 70.4 & 70.6 \\
\hline $\mathrm{PET} / \mathrm{CT}$ & 8 & 6 & 15 & 5 & 64.5 & 71.4 & 57.1 & 75.0 & 67.6 \\
\hline
\end{tabular}

CT, computed tomography; MRI, magnetic resonance imaging; PET/CT, positron emission tomography/computed tomography; LN, lymph node; TP, true-positive; FP, false-positive; TN, true-negative; FN, false negative; PPV, positive predictive value; NPV, negative predictive value, FIGO, International Federation of Gynecology and Obstetrics.

IB were $37.5 \%$ and $88.8 \%$ for $\mathrm{CT} ; 16.7 \%$ and $97.1 \%$ for MRI; and $41.7 \%$ and $91.8 \%$ for $\mathrm{PET} / \mathrm{CT}$, respectively. The sensitivities of three imaging modalities were lower than those of overall population. The difference of sensitivity between MRI and PET/ CT was statistically significant $(p=0.031)$. The differences of sensitivity were not statistically significant between CT and MRI ( $p=0.125$ ) as well as CT and PET/CT ( $p=1.000)$, respectively. The sensitivity and specificity of patients with FIGO IIA and IIB were 76.9\% and 61.9\% for CT; 38.5\% and 90.5\% for $\mathrm{MRI}$; and $64.5 \%$ and $71.4 \%$ for $\mathrm{PET} / \mathrm{CT}$, respectively. The sensitivities of three imaging modalities were higher than those of overall population. The differences of sensitivity among three imaging modalities were not statistically significant ( $p=0.125$ for $C T$ and $M R I, p=0.625$ for $C T$ and $\mathrm{PET} / \mathrm{CT}$, and $p=0.25$ for $\mathrm{MRI}$ and $\mathrm{PET} / \mathrm{CT}$, respectively).

\section{Discussion and Conclusion}

The purpose of this study was to assess the diagnostic accuracy of the three currently used imaging modalities, $C T, M R I$, and PET/CT, in evaluating pelvic LN involvement influencing treatment modality and prognosis.

The sensitivity and specificity of CT for detecting LN metastases from cervical cancer are reported to be $42.0 \%-$ $64.7 \%$ and $82.0 \%-96.6 \%$, respectively $[8,10]$. In our study, the sensitivity and specificity for LN sites were $51.4 \%$ and $85.9 \%$, respectively, and are similar to those reported by others.
The sensitivity and specificity of MRI for detecting LN metastases from cervical cancer are reported to be 30.3\%$70.6 \%$ and $74.0 \%-92.6 \%$, respectively $[8,10,11]$. In our study, the sensitivity and specificity for LN sites were $24.3 \%$ and $96.3 \%$, respectively. The current data showed that the sensitivity of MRI was relatively low for detection of metastatic LNs compared to those reported by others.

The sensitivity and specificity of PET/CT for detecting LN metastases from cervical cancer are reported to be $51.1 \%$ 75\% and 92.6\%-99.8\%, respectively [11-14]. In the present study, the sensitivity and specificity for LN sites were $48.6 \%$ and $89.5 \%$, respectively. The current data showed that the sensitivity and specificity of PET/CT were relatively low for detection of metastatic LNs compared to those reported by others.

The first possible reason for low-to-moderate sensitivities of CT, MRI and PET/CT in our study is that most patients (75.4\%) were of FIGO stage IA1 to IB1. In a study of 43 patients with stage IA-IIB uterine cervical cancer, Yang et al. [8] reported sensitivities with CT of $64.7 \%$ and MRI of $70.6 \%$ for pelvic $L N$ metastases when dividing per hemi-pelvis. Despite the similar study design based on hemi-pelvis, the higher sensitivity compared with our results is probably related to different distributions of FIGO stage. Approximately $28 \%$ of these patients were of FIGO stage IIA and IIB, whereas there were $15 \%$ with FIGO stage IIA and IIB in the current study. The different distribution of patients according to FIGO stage 
may lead to difference in detecting LN. Several studies have shown that imaging modalities have little value in detecting LN metastases in early-stage cervical cancer. Signorelli et al. [15] reported that the sensitivity rate was 32\% for PET/CT with FIGO stage IB1-IIA $<4 \mathrm{~cm}$ in diameter. Chou et al. [16] reported that FDG-PET detected only one (10\%) of the 10 pelvic LN metastases in patients with FIGO stage IA2 to IIA and MRIdefined negative nodal metastasis. Driscoll et al. [17] reported that the sensitivity rate was $0 \%$ for PET/CT in patients with early stage (FIGO stage IA to IB1) and MRI-defined LN-negative cervical cancer. In the present study, the sensitivities of three imaging modalities in patients with FIGO IA-IB were relatively low compared to those of patients with FIGO IIA-IIB. As for $M R I$, the use of only size criteria could be the additional reason for the relatively low sensitivity. Although the diagnostic accuracy of detecting LN metastasis by MRI is dependent on the LN size and LN sizes greater than $1 \mathrm{~cm}$ in the short axis have been generally considered abnormal, the use of only size criteria on MRI has been proven to be of limited diagnostic accuracy and this criteria may have resulted in a relatively low sensitivity. It could be difficult to differentiate between nonmetastatic and metastatic LNs of similar size and normal sized LNs may also be involved with metastatic disease. Several factors have been suggested to improve the sensitivity of MRI. First, the diagnostic accuracy of MRI could be improved when combined with morphologic parameters. Choi et al. [18] reported that lobulated or spiculated LN margins were strong predictors of LN metastasis. Yang et al. [8] reported that central necrosis is useful in the detection of metastasis in pelvic LNs in uterine cervical cancer. Second, the ADC based on diffusion-weighted imaging (DWI) is a relatively independent modality of lesion size for evaluation of LN metastases. DWI has been used for the differentiation between clinically benign and malignant lesions in extra-cranial organs. Kim et al. [19] reported that DWI was useful in detecting metastatic LNs in uterine cervical cancer. Choi et al. [20] reported that a node-by-node comparison of DWI improved the diagnostic performance compared with conventional size-based criteria on T2-weighted imaging in patients with uterine cervical cancer.

A number of factors could explain the discrepancies of PET/CT between our study and previous ones. First, FDG is not a cancer-specific agent, and inflammatory cells such as macrophages and neutrophil in areas of infection or inflammation will also accumulate FDG $[21,22]$. FDG-PET/CT has still a difficulty in differentiating between inflammatory and metastatic LNs. Additionally, multiple structures with variable physiologic uptake of FDG may be seen in the abdomen and pelvis. The ovaries containing functional cysts may lead to increased FDG activity mimicking LN metastases, and normal ovarian uptake may be misinterpreted as a metastatic lesion $[23,24]$. Second, the uptake value of PET/CT is unsatisfactory when metastatic LNs are small sized. Kitajima et al. [25] found that PET/CT had a detection sensitivity of $16.7 \%$ in metastatic LNs with a short-axis diameter of $4 \mathrm{~mm}$ or less. This limited sensitivity is based chiefly on insufficient detection of small metastatic LNs. We suggest that the diagnostic accuracy of PET/CT could be improved when combined with morphologic findings provided by MRI. Kim et al. [26] reported that fused MR/PET imaging compared with PET/CT is suggested to be useful for detecting metastatic LNs in uterine cervical cancer.

Taken together, CT, MRI, and PET/CT in our patients with uterine cervical cancers have relatively low to moderate sensitivity and moderate to high specificity for detecting pelvic metastatic LNs compared to those reported by others. The discrepancy could be explained that we performed histopathologic examination of LNs with slices made at $2 \mathrm{~mm}$ thickness, whereas LNs are usually evaluated in only 1 or 2 parallel sections. Lentz et al. [27] reported that micrometastases was identified in histologically negative LNs in 19 patients (15\%) of early-stage cervical cancer. Therefore, it is possible that pelvic LN metastasis may have been underestimated with the conventional techniques.

Although the current data showed that neither CT/MRI nor PET/CT is perfect for detecting LN metastases, the imaging modalities may be helpful for determining of treatment field and dose for boost EBRT in uterine cervical cancer. According to the National Comprehensive Cancer Network (NCCN) guidelines, highly conformal boosts of an additional 10-15 Gy may be considered for limited volumes of gross unresected lymphadenopathy. Several studies have used imaging modalities to determine the boost field in uterine cervical cancer. Ariga et al. [5] reported that the boost EBRT was delivered to clinically metastatic pelvic LNs assessed by CT/ MRI. Yoon et al. [28] reported that the involved LNs detected on pre-RT PET/CT were treated with doses from 59.4 Gy to 63.0 Gy, with an additional boost dose of 5.4 to 9.0 Gy delivered to the residual LNs seen on PET/CT taken immediately after the completion of RT.

The current study had some limitations. First, this study was a retrospective study with small sample size. Second, only one radiologist and nuclear medicine physician read each image from CT, MRI, and PET/CT. The images should be interpreted 
independently by at least two researchers to ensure objectivity and to reduce inter-personal variation. Third, because of retrospective design, histopathological classification of pelvic LNs was not grouped into regions-specific categories. For this reason, all individual LNs detected on imaging could not be correlated to the surgically removed LNs based on nodal regions-specific comparison. Although the hemi-pelvis based classification was applied to several other studies of uterine neoplasms $[8,9]$, we believe that the node-by-node correlation should be carried out in a future study.

In conclusion, the results of our analysis showed that preoperative $\mathrm{CT}, \mathrm{MRI}$, and $\mathrm{PET} / \mathrm{CT}$ had low to moderate sensitivity and PPV, and moderate to high specificity, NPV, and accuracy. CT and PET/CT are more useful for detection of pelvic LN metastases than MRI; although CT and PET/CT still had a very low sensitivity. More efforts are necessary to improve sensitivity of imaging modalities in order to predict pelvic LN metastases in patients who will receive definitive chemoradiotherapy without surgery for uterine cervical cancer.

\section{Conflict of Interest}

No potential conflict of interest relevant to this article was reported.

\section{References}

1. Oh CM, Won YJ, Jung KW, et al. Cancer Statistics in Korea: Incidence, Mortality, Survival, and Prevalence in 2013. Cancer Res Treat 2016;48:436-50.

2. Kang JK, Kim MS, Jang WI, et al. The clinical utilization of radiation therapy in Korea between 2009 and 2013. Radiat Oncol J 2016;34:88-95.

3. Kidd EA, Siegel BA, Dehdashti $F$, et al. Lymph node staging by positron emission tomography in cervical cancer: relationship to prognosis. J Clin Oncol 2010;28:2108-13.

4. Inoue $T$, Morita $K$. The prognostic significance of number of positive nodes in cervical carcinoma stages IB, IIA, and IIB. Cancer 1990;65:1923-7.

5. Ariga T, Toita T, Kasuya G, et al. External beam boost irradiation for clinically positive pelvic nodes in patients with uterine cervical cancer. J Radiat Res 2013;54:690-6.

6. Chan JK, Cheung MK, Huh WK, et al. Therapeutic role of lymph node resection in endometrioid corpus cancer: a study of 12,333 patients. Cancer 2006;107:1823-30.

7. Benedetti Panici P, Basile S, Angioli R. Pelvic and aortic lymphadenectomy in cervical cancer: the standardization of surgical procedure and its clinical impact. Gynecol Oncol 2009;113:284-90.

8. Yang WT, Lam WW, Yu MY, Cheung TH, Metreweli C. Comparison of dynamic helical CT and dynamic MR imaging in the evaluation of pelvic lymph nodes in cervical carcinoma. AJR Am J Roentgenol 2000;175:759-66.

9. Horowitz NS, Dehdashti F, Herzog TJ, et al. Prospective evaluation of FDG-PET for detecting pelvic and para-aortic lymph node metastasis in uterine corpus cancer. Gynecol Oncol 2004;95:546-51.

10. Horowitz NS, Dehdashti F, Herzog TJ, et al. Prospective evaluation of FDG-PET for detecting pelvic and para-aortic lymph node metastasis in uterine corpus cancer. Gynecol Oncol 2004;95:546-51.

11. Choi HJ, Roh JW, Seo SS, et al. Comparison of the accuracy of magnetic resonance imaging and positron emission tomography/computed tomography in the presurgical detection of lymph node metastases in patients with uterine cervical carcinoma: a prospective study. Cancer 2006;106:91422.

12. Sironi $S$, Buda $A$, Picchio $M$, et al. Lymph node metastasis in patients with clinical early-stage cervical cancer: detection with integrated FDG PET/CT. Radiology 2006;238:272-9.

13. Kitajima K, Murakami K, Yamasaki E, Kaji Y, Sugimura K. Accuracy of integrated FDG-PET/contrast-enhanced CT in detecting pelvic and paraaortic lymph node metastasis in patients with uterine cancer. Eur Radiol 2009;19:1529-36.

14. Loft $A$, Berthelsen $A K$, Roed $H$, et al. The diagnostic value of $\mathrm{PET} / \mathrm{CT}$ scanning in patients with cervical cancer: a prospective study. Gynecol Oncol 2007;106:29-34.

15. Signorelli M, Guerra L, Montanelli L, et al. Preoperative staging of cervical cancer: is 18-FDG-PET/CT really effective in patients with early stage disease? Gynecol Oncol 2011;123:236-40.

16. Chou HH, Chang TC, Yen TC, et al. Low value of [18F]fluoro-2-deoxy-D-glucose positron emission tomography in primary staging of early-stage cervical cancer before radical hysterectomy. J Clin Oncol 2006;24:123-8.

17. Driscoll DO, Halpenny D, Johnston C, Sheehy $N$, Keogan M. 18F-FDG-PET/CT is of limited value in primary staging of early stage cervical cancer. Abdom Imaging 2015;40:127-33.

18. Choi HJ, Kim SH, Seo SS, et al. MRI for pretreatment lymph node staging in uterine cervical cancer. AJR Am J Roentgenol 2006;187:W538-43.

19. Kim JK, Kim KA, Park BW, Kim N, Cho KS. Feasibility of diffusion-weighted imaging in the differentiation of metastatic from nonmetastatic lymph nodes: early experience. J Magn Reson Imaging 2008;28:714-9. 
20. Choi EK, Kim JK, Choi HJ, et al. Node-by-node correlation between MR and PET/CT in patients with uterine cervical cancer: diffusion-weighted imaging versus size-based criteria on T2WI. Eur Radiol 2009;19:2024-32.

21. Alavi A, Gupta N, Alberini JL, et al. Positron emission tomography imaging in nonmalignant thoracic disorders. Semin Nucl Med 2002;32:293-321.

22. Carter KR, Kotlyarov E. Common causes of false positive F18 FDG PET/CT scans in oncology. Braz Arch Biol Technol 2007;50(SPE):29-35.

23. Ames J, Blodgett T, Meltzer C. 18F-FDG uptake in an ovary containing a hemorrhagic corpus luteal cyst: false-positive PET/CT in a patient with cervical carcinoma. AJR Am J Roentgenol 2005;185:1057-9.

24. Yildirim Y, Sehirali $S$, Avci ME, et al. Integrated PET/CT for the evaluation of para-aortic nodal metastasis in locally advanced cervical cancer patients with negative conventional CT findings. Gynecol Oncol 2008;108:154-9.
25. Kitajima K, Murakami K, Yamasaki E, et al. Accuracy of 18F-FDG PET/CT in detecting pelvic and paraaortic lymph node metastasis in patients with endometrial cancer. AJR Am J Roentgenol 2008;190:1652-8.

26. Kim SK, Choi HJ, Park SY, et al. Additional value of MR/PET fusion compared with PET/CT in the detection of lymph node metastases in cervical cancer patients. Eur J Cancer 2009;45:2103-9.

27. Lentz SE, Muderspach LI, Felix JC, Ye W, Groshen S, Amezcua CA. Identification of micrometastases in histologically negative lymph nodes of early-stage cervical cancer patients. Obstet Gynecol 2004;103:1204-10.

28. Yoon MS, Ahn SJ, Nah BS, et al. Metabolic response of lymph nodes immediately after RT is related with survival outcome of patients with pelvic node-positive cervical cancer using consecutive [18F]fluorodeoxyglucose-positron emission tomography/computed tomography. Int J Radiat Oncol Biol Phys 2012;84:e491-7. 